\title{
TOWARDS AN ALTERNATIVE BLUEPRINT FOR A PHYSICALLY-BASED DIGITALLY SIMULATED HYDROLOGIC RESPONSE MODELLING SYSTEM
}

\author{
Keith Beven \\ Institute of Environmental and Natural Sciences \\ Lancaster University, Lancaster LA1 4YQ, UK
}

\begin{abstract}
"We always reason correctly when we apply to an object only ideas extracted from its own nature; but, on the contrary, we fall in all kinds of errors in persisting to conclude before knowing, and to know before having examined..... When the object is material and, ignoring the size and form of its elementary parts, as well as the laws that the Author of Nature has prescribed for them, we want nonetheless to foresee the effects, calculate the stresses, direct the actions; then Nature manifests itself independent with respect to us and, always faithful to the law that has been prescribed to it but that we ignore, opposes our conceptions, confounds our prospects, thwarts our efforts......To question Nature, to study the laws she has dictated for herself, to catch her in the fact, to steal her secret, is the only means to dominate her and the true way of every reasonable spirit."
\end{abstract}

Pierre du Buat, 1786,

Principles d'hydraulique, vérifiés par un grand nombre d'expériences faites par ordre du gouvernement. Ouvrage dans lequel on traite du mouvement uniforme et varié dans les Rivières, les Canaux et les Tuyaux de conduite; de l'origine des Fleuves, .... de la résistance des Fluides en générale; \& de celle de l'Air \& de L'Eau en particulier (quoted in Levi, 1995)

"Every theory is based on physical concepts expressed through mathematical idealisations. They are introduced to give an adequate representation of the physical phenomena. No physical concept is sufficiently defined without the knowledge of its domain of validity."

Leon Rosenfeld (quoted in Prigogine, 1997)

"We must concede that our paper is more of an "artist's conception" than a true Blueprint."

Freeze and Harlan, 1969

Keywords: Distributed hydrological modelling, conservation equations, landscape, model space, mapping, uniqueness 


\section{Introduction}

It is now 30 years since the seminal Blueprint paper of Freeze and Harlan (1969, FH69) first set out the equations and boundary conditions for a "physically-based digitally simulated hydrologic response model". It therefore seems timely to briefly review the progress that has been made in hydrological modelling and to examine whether an Alternative Blueprint should be considered as the basis for hydrological modelling at the catchment scale.

What has happened in that 30 years? There are certainly now a variety of models that are essentially based on the FH69 Blueprint but there has also been a continuing widespread use of the type of lumped conceptual models for discharge prediction, revitalised recently by the type of data-based transfer function models used, for example, by Jakeman and Hornberger (1993) and Young and Beven (1994). The modern transfer function approach is at the opposite end of the modelling spectrum to the FH69 Blueprint in that the aim is to let the data define an appropriate model structure, rather than to use process theory to define a structure a priori as in the Blueprint. For some purposes (such as adaptive real time forecasting) there would not appear to be any reason to use more complex modelling approaches.

There has also been the development of a number of simplified theoretical structures of which perhaps the most widely used is TOPMODEL (Beven and Kirkby, 1979; Beven et al., 1995; Beven, 1997) which, although it is computationally simple, has the capability of producing distributed predictions of catchment storage or water table levels (see for example Ambroise et al., 1996; Lamb et al., 1997; Seibert, 1997). Relative to the FH69 Blueprint, TOPMODEL makes strong simplifying assumptions that will not be appropriate in many catchments (see for example Barling et al., 1994). Some of these assumptions can be relaxed (see critique in Beven, 1997, and Beven and Freer, 2000a) but the user has to take care that the assumptions are not invalidated in any application. These models are not necessarily general in their applicability.

In their Blueprint paper, Freeze and Harlan aimed to provide a structure that was generally applicable. They wrote down the equations for different surface and 
subsurface flow processes and showed how they could be linked by means of common boundary conditions into a single modelling framework. Their analysis is still the basis of the most advanced distributed rainfall-runoff modelling systems today (e.g. Abbott and Refsgaard, 1996). However, the descriptive equations that they used for each process also required, in all cases, certain simplifying assumptions. Thus, for subsurface flow, it is assumed that both saturated and unsaturated flows can be described by Darcy's law, while for surface flows it was assumed that the flow could be treated as a onedimensional, cross-section averaged flow either downslope over the surface or along a reach of the channel network in a catchment (leading to the St. Venant equations).

In 1969, Freeze and Harlan concluded that the level of computing power available at that time would allow treatment of

- one- and two-dimensional transient soil-moisture flow in heterogeneous soils, with three-dimensional numerical solutions imminent

- three-dimensional, steady-state and two-dimensional transient groundwater flow in heterogeneous, anisotropic porous media

- one-dimensional, unsteady, non-uniform, spatially-varied open-channel flow with lateral inflow and local channel infiltration.

They suggested that further research was required on controls on evapotranspiration processes, the continuity between saturated and unsaturated flow, the continuity in flow between confined and unconfined aquifers, non-steady channel flow in irregular natural channels and the role of vegetation in hydrological processes.

This type of distributed model allows the prediction of local hydrological responses for points within the catchment. The first applications of this type of model were made for hypothetical catchments and hillslopes by Freeze (1972). The calculations required the largest computers available at the time (Al Freeze was then working at the IBM Thomas J. Watson Research Centre at Yorktown Heights), and even then only a limited flow domain and coarse mesh of points could be solved. The first application to a field site of this type of model was published by Stephenson and Freeze (1974) who attempted to 
model a single hillslope at the Sleepers River catchment in Idaho. The results were not particularly successful but, as they pointed out, it was a complex hillslope underlain by fractured basalts, with complex flow pathways and limited knowledge of the inputs and initial conditions for the simulation. In addition, computing constraints limited the number of simulations they could actually try. Arising from these difficulties, they were also the amongst the first to discuss the difficulties of validation of hydrological models.

There has been a strong surge in the use of distributed modelling based on this Blueprint over the last decade. This has partly been because the increase in computer power, programming tools and digital databases has made the development and use of such models so much easier and partly because there is a natural tendency for a model development team to try to build in as much understanding from their perceptual model of the important processes as possible. Thus, there is an obvious attraction of distributed process modelling. There are also very good scientific reasons underlying the effort. One is the need for distributed predictions of flow pathways as a basis for other types of modelling, such as the transport of sediments or contaminants. Another is the need for predictions of the impacts of land use and other changes, when it is argued that this will only be possible with physically-based models.

Examples of distributed physically-based models include the SHE model Système Hydrologique Européen (e.g. Abbott et al., 1986) and the SHETRAN and MIKE SHE variants (Bathurst et al., 1995; Parkin et al., 1996; Refsgaard and Storm, 1995; Refsgaard, 1997), the IHDM model (Institute of Hydrology Distributed Model, e.g. Calver and Wood, 1995), in Australia there is the CSIRO TOPOG model (e.g. Vertessy et al. 1993), in Germany HILLFLOW (Bronstert and Plate, 1997) and there are a number of others. They differ primarily in the way they discretise a catchment and solve the process equations (sometimes with simplifications), but all are essentially based on the original FH69 Blueprint as a description of the flow processes. Advances in computer power have also now greatly relaxed the limitations on the solutions possible. Fully three-dimensional subsurface flow solutions to coupled two-dimensional surface solutions are now possible on desktop workstations and parallel workstations will allow finer element meshes and larger catchments to be simulated. Increased graphic capabilities have also made the preparation of spatial datafiles and the presentation of model results much easier. It is worth asking, however, whether there might be 
alternative ways of using the available computer power in the next millennium since we must recognise a fundamental difference between solving the flow equations in hydrology and other fluid dynamics disciplines such as meteorology, oceanography and limnology. This difference is that in surface and subsurface hydrology the small scale flows are largely dominated by the local geometry and local boundary resistances of the individual flow pathways rather than the dynamics of the fluid itself. This means that computational improvements are not only a matter of resolving finer characteristics of the flow but also of characterising of the medium or channels in which the flow is taking place (Beven, 2000). This is not a computational problem (but interacts with the computational problem in terms of the effective parameter values required for the solution).

The hydrological literature is not short of reviews and discussions of the advantages, limitations and potential of physically-based distributed models. Some interesting examples are the papers of Beven (1989); Grayson et al.(1992); Smith et al. (1994); De Marsily (1994); Woolhiser (1996); and the exchange between Beven (1996) and Refsgaard et al. (1996). It is not necessary to rehearse all the arguments here but I will concentrate on the question of whether it might be possible to improve on the modelling strategy embodied in the FH69 Blueprint. The ideas presented are deliberately radical with the aim of stimulating discussion. I do not expect, however, that this Alternative Blueprint will define the modelling agenda for the next 30 years, since ultimately advances in this field will be far more dependent on the development of new measurement techniques or more creative use of existing measurement techniques than on new theory or computational techniques. What is offered here is more of a modelling methodology that can be applied to a variety of potential existing and future modelling structures.

\section{Why does the FH69 Blueprint need to be reconsidered?}

There is one very important limitation of the FH69 Blueprint that will ultimately result in it being abandoned. Particularly in its description of unsaturated subsurface flow, it is based on Darcian theory that may be applicable at small scales but is certainly not applicable at large scales due to the effects of the nonlinearity of the Darcy flow equation, the heterogeneity of soil properties and preferential flow of different types. 
Models that derive from the FH69 Blueprint have, with some few exceptions, not attempted to change the simplified process description that underlies it. There is thus a very large investment in structures that are based on a rigorous but inappropriate description of the flow processes, but that assume that the theory can be used if "effective" parameter values can be found. That the concept of "effective" parameter values is unsound was demonstrated over a decade ago by Binley et al. (1989). This alone should induce a re-evaluation of models based on the Darcian Blueprint and perhaps would have done so already if an obvious alternative theory existed to put in its place.

What are the prospects for an improved theory? In one sense a complete process understanding already exists in the form of the Navier-Stokes equations that are the basis of a detailed description of all fluid flows. The difficulty in modelling hydrologicallyrelevant flows is not that the underlying physical basis is not well understood at small scales, it is that the boundary conditions for the flows are so variable that integration to a larger hydrological scale is both a theoretical and practical constraint to the application of the Navier-Stokes description (even if computational constraints are ignored). A recent attempt at this, invoking mass, energy and momentum conservation balances and entropy constraints at larger scales has been provided by Reggiani et al. (1998, 1999, 2000, see below).

Thus, the Darcian Blueprint can already be considered as an approximation to a fuller physical description that is an attempt at an integration over a certain population of pore spaces. The problem of heterogeneity of pore space boundary conditions is not, however, eliminated at the Darcian scale; a problem that has long been recognised as heterogeneity in the soil hydraulic properties. There have certainly been some attempts to take account of heterogeneity of porous medium properties, including fractal heterogeneity (e.g. Neuman, 1995; Pachepsky and Timlin, 1998)and to include preferential flows into hillslope and catchment scale models (e.g. Bronstert and Plate, 1997; Faeh et al., 1997). There would appear to be a very fundamental limitation to such attempts, however, due to critical measurement limitations and the essential scale dependent nature of any such representations (as recognised by Beven, 1995, and explicitly in the theory of Reggiani et al., 1999). 
Catchment hydrology suffers from a measurement problem in that, with the exception of discharge measurements, most other measurement techniques provide information at small scales (and are also often invasive with the potential to change the response of the system by the very process of observation). This is a basic limitation on the possibility for characterising a catchment system, and would remain so even if the FH69 Darcian Blueprint were correct or if some revised theory of flow in heterogeneous soils with preferential flows (which would undoubtedly have more parameters to be determined) could be constructed.

Thus, although there are important limitations in the basic theory of the current Blueprint, the far greater problem is in the proper characterisation of the uniqueness of individual catchments. There is evidence for this in the fact that 30 years after FH69 there are still very few applications of physically-based models based on measured or estimated parameters alone and very few validations of distributed predictions against distributed measurements. In the whole of the recent book on Distributed Modelling edited by Abbott and Refsgaard (1996), for example, there appears to be only one comparison between predictions of a distributed model and internal state measurements in a model of the Karup catchment in Denmark for which three point water table measurements are compared with predictions on a $500 \mathrm{~m}$ grid (p135). The comparison shows that there is significant uncertainty in the predictions. Parkin et al. (1996) in another application of the SHE model to the small Rimbaud catchment in the south of France, demonstrate considerable uncertainty in discharge predictions based only on prior estimates of effective parameter values, conditioned on a few point field measurements. Loague and Kiryakidis (1997) report on the latest, not very successful, attempts to model the R-5 catchment at Coshocton using only measured, spatially variable, infiltration parameters, concluding that, despite all the work that has been done at the site, their process representation is not adequate.

There is an argument, proffered, for example, by De Marsily (1994) and Smith et al. (1994) that a proper understanding of the nature and causes of the heterogeneity of soil and aquifer parameter values should lead to improvements in physically-based modelling. In this view, the limitations inherent in current applications does not invalidate the basis of the (FH69) equations of such models (The Type 1 models of Smith et al., 1994). While this might be considered to be self-evidently true, the potential for 
improvement in this way must necessarily be limited. Understanding of the nature and causes of heterogeneity of characteristics necessarily introduces complexity.

Complexity almost inevitably introduces more parameter values in any description of that complexity. Requiring more parameter values implies more measurements that must be made to define those parameters, even if only to define the nature of the stochastic distribution being used to describe the heterogeneity. And if the distributions are likely themselves to be spatially heterogeneous with horizon and position in the catchment it is difficult to see that such a characterisation will ever be practically feasible with the point measurement techniques currently available, or at least not without considerable uncertainty. This would not, therefore, appear to be a satisfactory solution to the problems of the current Blueprint.

The only chance of success would be if detailed studies in some locations could be used to define the characteristics of other locations on the basis of simpler measurements in some way. One example strategy is the use of soil texture variables in the regression relationships for soil hydraulic characteristics known as pedotransfer functions. These currently ignore heterogeneity, often even ignore the standard errors of estimation, and any more parameter rich approaches must introduce considerable uncertainty in applications to specific catchments with their own unique characteristics. Experience, such as in the R-5 catchment mentioned above, suggests that the prognosis for the survival of the current Blueprint by this cure is not good.

\section{Towards an Alternative Blueprint at hydrological relevant scales}

The FH69 Blueprint is an approach based on the aggregation of (already approximate) small scale theory to larger scale applications. It serves, in essence, as a scaling theory, using the partial differential equations as a way of extrapolating limited small scale knowledge to the scale of hillslopes and catchments. The Alternative Blueprint to be proposed here takes a very different approach based on disaggregation of the hydrological functioning of different parts of the landscape, which is, after all, what we are interested in (after the precursory requirement of using models to formulate our hydrological knowledge). 
There are two major steps in this Alternative Blueprint. The first is to define a "model space" that is sufficiently broad so as to encompass the range of functional responses within the catchment of interest (noting that the current generation of physically-based models might be one contender as a description of the functional responses). The second is to map the "landscape space" of the catchment into this model space on the basis of all the information that is available about the catchment. Because of uncertainties in both model structures, boundary conditions and observations, this will necessarily be an uncertain or fuzzy mapping, resulting in predictive uncertainty (Beven, 2000). There is also the possibility of a third stage in which the model space is reduced to a number of functional classes to facilitate the computation of predictions (see for example Franks and Beven, 1998, Beven and Franks, 1999).

What then is this "model space"? There is no unambiguous definition of assumptions and equations (as in the FH69 Blueprint) since there is no unambiguous description of catchment flow processes given normal levels of knowledge of a catchment, even at research sites. The model space may, a priori, include a number of different contending model structures and within each structure a variety of parameter sets that are expected to give reasonable predictions of the functional response of the catchment or points within the catchment. The model space explicitly recognises that it may not be possible to specify a single model structure or parameter set as the "correct" description of the catchment (see following section). It may be visualised as a high dimensional space in which potential model structures and associated parameter values form the axes (figure 1). The only critical constraint at this point is that the models considered must make predictions of the variables required in an application and of the variables for which observations are available for model evaluation (see below).

The most important component of this Alternative Blueprint is then the mapping of the (unique) catchment under study (the landscape space) into this model space. This is treated as a conditioning process by evaluating different models (structures and parameter sets) in terms of how well they reproduce the observations. Some models will not reproduce the observations and can be rejected as "non-behavioural". Others will reproduce the observations to some agreed level of acceptability and will be retained as "behavioural". However, there may be no clear boundary between what is considered acceptable and what is not and many models may fall into a middle ground of possibly 
acceptable. This mapping must therefore necessarily be a fuzzy mapping. This should not be considered a disadvantage of the proposed methodology, but rather an explicit recognition within the Alternative Blueprint of the real nature of the modelling process. It is the idea that there can be a true model of the reality that is a unique catchment with our current state of knowledge and measurement techniques that, while a commendable aim, is in practice artificial.

The problems of evaluating acceptability and deciding what is an appropriate level of acceptability will be discussed below. However, it is worth noting here that this Blueprint replaces a need for the calibration of parameter values in favour of the conditioning process that is the fuzzy mapping into the model space.

\section{Defining the model space: the conservation of mass}

Consider the local mass balance equation which, in the continuum form, may be stated as:

$$
\frac{d S}{d t}=\nabla q+p-e
$$

where $S$ is a local mass storage, $\nabla q$ is the divergence in local mass flux, $p$ is a local source term (such as precipitation) and e is a local loss term (such as evapotranspiration). It is worth noting in passing that this self-evident law is not generally verifiable in any hydrological system of practical interest, due to unknown measurement errors in all the variables and lack of knowledge of the boundary conditions, particularly for subsurface flow components. It is, however, an appropriate constraint on any hydrological model. Other conservation equations (for energy or momentum, Reggiani et al., 1998, 1999) are equally general but cannot generally be used as model constraints since energy losses are extremely difficult to formulate in other than very special conditions (see below).

The mass balance equation is not, however, particularly useful in this local form. Digital computers and practical hydrological models necessarily work in discrete space and time increments, whether the spatial discretisation be at the scale of catchments, sub- 
catchments, hydrological response units or at the scale of finite elements or finite volumes representing discretised soil profiles or hillslope. Process descriptions based on continuum mechanics are not necessarily advantageous in this discrete space. Following Reggiani et al. $(1998,1999)$ a control volume form of (1) will be generally more useful as:

$$
\iiint \int d S d V d t=\int \oint q d x d t \pm \iiint \int e d V d t
$$

where $q$ is now a volume flux normal to the boundary (including precipitation inputs), $e$ is now a source or sink term within the volume and $\oint d x$ denotes an integration over the external surfaces of the control volume. This equation is the basis for all hydrological models and may be applied regardless of process, scale and discretisation. It is not, in itself, a sufficient basis for defining a hydrological model. Additional relationships are required for the dependence of all the variables on the right hand side with both local storage $S$ and, if necessary, the storages in adjacent connected volumes (this might not be necessary if the control volume is at the catchment scale and the catchment is considered to have an impermeable base).

These dependencies can be separated into two (related) parts: intra-volume and intervolume dependencies. Intra-volume dependencies will reflect the effects of the distribution of water within the element on the variables, $q$ and e. Inter-volume dependencies will reflect the effects of the distribution of water within adjacent elements on the boundary fluxes, q. For the case of subsurface flow, the FH69 Blueprint used an average potential (or moisture content) and the soil water characteristic curves to define the intra-volume dependencies, and a first order potential gradient relationship (Darcy's law) to define the inter-volume dependencies.

This choices are not necessarily self-evident and, as pointed out above, may be a misapplication of a small scale description at larger scales. This is understandable, given the lack of larger scale measurement techniques that might allow a more appropriate description, but we should consider alternative choices in an attempt to produce a more realistic (if probably not verifiable) description. 
For control volumes at larger scales, it must be recognised that within the volume there is no single potential status for the water stored within it. There is, rather, a distribution of potentials within the volume associated with local storages and dependent on local interconnections. Adding water to any control volume will, therefore, not simply change the average water potential but will cause a redistribution of water within the volume over a certain relaxation time. The different intra-volume storage states may, in fact, be competing for parts of the total storage capacity (as in the unsaturated and saturated components of the hillslope control volume of Duffy, 1996). In general, the changes induced will be nonlinear with respect to both the initial distribution of storage and the rate at which water is added.

This has long been recognised in the form of the hysteresis commonly observed in soil moisture characteristics, even in small samples (and commonly neglected in "physicallybased" models of soil water). Various simplified models of hysteresis may be found in the soil physics literature (e.g. Jaynes, 1990), none of which adequately characterise the variety of process mechanisms and time scales that might be involved. A critical view would suggest that hysteresis, while accepted as a "physically-based" concept, is a desperate attempt to rescue a soil physics that is already an inadequate description of the effects of structure and connectivity on the relationship between flow and storage in real soils, even at small scales.

What then are the implications for a "physically-based" description at the scale of a practical control volume? Clearly, any intra-volume changes in storage in response to changes in boundary fluxes are going to be complex, more complex than those causing the observed hysteresis in small samples. Additional heterogeneity of inputs and soil characteristics, preferential flow pathways, differences of local wave speeds relative to flow velocities, distributions of active roots etc. will be involved, while distances and time scales for the redistribution processes will be greater. A simple Darcian, or hysteretic Darcian description can be considered as one possible way of defining the nature of the fluxes on the basis of a simplified "physics" assuming a single intra-volume storage state.

The simplest, first order, means of closing the dependencies of equation (2) is to assume that all the fluxes are a function of storage alone. A unique relationship 
between storage and flux leads to the kinematic wave equation that has been widely used as an approximation in hydrological modelling for both surface and subsurface processes (Eagleson, 1970; Singh, 1996; Beven, 2001). However, as for the mass balance equation itself, neither storage nor flux can generally be measured directly so the adequacy or inadequacy of such a description can only be inferred indirectly on the basis of predictions of observable quantities such as catchment discharge. Such predictions will, however, require the specification of both boundary conditions and parameter values which also cannot be measured directly or estimated on the basis of theoretical reasoning. Thus, even with such a first order description there is a necessary dependence on the circular process of parameter calibration and more "complete" descriptions (for example, accounting for the time variable "hysteretic" dependence of fluxes on storage in the control volume) will have a greater dependence on calibration.

Thus, there are in fact two problems to be solved in the application of (2) in any Alternative Blueprint. The first is the nature of the intra- and inter-volume dependencies; the second is the calibration of the parameter values given a certain set of observables. For the present, we will assume that (2) may be written as

$$
\iiint \int d S d V d t=\int \oint q(\underline{S}) d x d t \pm \iiint \int e(\underline{S}) d V d t
$$

where $(\underline{S})$ represents a dependence on a set of storage states in both space (including intra-volume states) and earlier times. We note that, at the current time and at control volume scales of hydrological interest, $\mathrm{S}$ cannot be generally considered an observable, nor can any intra-volume storage states other than at local point scales. This clearly has important implications for both the definition of a physically-based model structure and for the identification of any parameter values involved in the definition.

\section{Defining the model space: the use of a conservation of energy principle}

The obvious additional physical principle to invoke at this point is the conservation of energy (e.g. Reggiani et al., 1998). The primary driving forces for hydrological systems are the distribution of potential energy of precipitation inputs as dominated by the catchment topography, and the inputs of energy due to radiation from the sun. As with 
storage, in principle an energy balance can be defined for any control volume in the system; in practice the energy states of the water are not directly observable at other than the point scale. Any such energy balance will inevitably involve some energy loss coefficients, to reflect the effects of friction and laminar and turbulent shear, that require knowledge of the geometry and characteristics of the flow paths that will generally not be available, except in the simplest cases. This has required in previous analyses, such as that of the FH69 Blueprint, that bulk energy loss coefficients be used in model definition (as in the uniform flow equations of channel flow, in the interpretation of the hydraulic conductivity as an inverse head loss coefficient, and in the canopy resistance coefficients of evapotranspiration models). More complex expositions are currently under development (e.g. Ewen, 1996; Germann and Di Pietro, 1999) but, as yet, are lacking in that they do not specify how the energy loss coefficients might generally be specified except by the circular process of back-calculation from measured fluxes (and then only for scales for which this is technically possible).

Model descriptions based on conservation of energy have been relatively successful for channelled surface flows, despite the fact that only an approximate energy balance accounting in terms of the total momentum of the flow is normally done. The accounting is implicit in the specification of an appropriate roughness coefficient in a specific form of velocity equation (and commonly ignores external energy sources as negligible). Such descriptions have also been relatively successful for well-connected saturated subsurface flows for which energy gradients are also relatively well defined. It has also been applied (usually in a totally uncritical way) to irregular overland flows (under a sheet flow assumption) and poorly connected flows in the unsaturated zone. In these cases, the approach does not (as yet) appear to provide an adequate answer to an Alternative Blueprint. In any control volume there could be a wide range of concurrent total energy states and it is simply too difficult to define the energy loss coefficients involved.

This is a further indication that deductive reasoning based on physical reasoning alone is not a sufficient means of defining a useful model of a complex catchment system. The need to reflect the unique nature of that catchment in terms of storage or energy loss coefficients necessarily requires consideration of what is also observable (Beven, 2000). 


\section{Defining the model space: the choice of an appropriate scale of control volume}

The classical Darcian description of subsurface flow has associated with it a lower scale limit for the continuum description, the Representative Elementary Volume (REV). This limit (often discussed but never, to my knowledge, precisely defined for a particular soil) is the scale at which an effective soil water potential within some local population of pore spaces is meaningful. It is therefore also closely related to the scale over which any average potential gradient might be meaningful. No equivalent concept for overland flows appears to have been formulated. It is not necessary if a simple average depth or storage-flow function assumption already ignores any real effects of the irregularity of depths and velocities within a flow, except in so far as they might have an effect on an effective roughness coefficient.

What is not often recognised is that, as well as this lower scale limit, there must also be an upper limit to the utility of an average potential and potential gradient due to the effects of soil or rock structure and heterogeneity. What work that has been done in this area has concentrated on trying to retain the concept of the average potential and gradient and to formulate a heterogeneity and scale dependent resistance coefficient by means of a statistical generalisation of the intra-volume heterogeneity (see for example Dagan for the case of saturated flows). There would appear to be two problems in applying the available theory. The first is that it requires very specific assumptions to be made about the nature of the heterogeneity. However, the equifinality problem may then arise again in terms of different parameterisations of those assumptions or different alternative sets of assumptions might give equally acceptable predictions. The second, and more important, is that the assumption of an average potential gradient as a control for fluxes between control volumes will have limited utility in situations where average potentials and potential gradients are rapidly varying within a control volume. This will generally be more significant in unsaturated flow than saturated flow, but even in saturated systems boreholes in close proximity may yield significantly different apparent piezometric heads as a result of poor hydraulic linkages or, perhaps, poor installation techniques.

At larger scales, therefore, if the concept of an average potential gradient is not physically meaningful, the only variable that might be used as the basis for a description 
is the storage in the control volume. This is an absolute physical quantity, which must be subject to the mass balance equation, but which will necessarily have a complex relationship, hysteretic and scale dependent, with the inter-volume fluxes. Use of control volume storage does not, in fact, arbitrarily restrict the discretisation scales that can be considered in a physically-based description. Even the catchment scale could be used if a proper scale dependent physically-based representation of the effects of intracatchment variability on boundary fluxes could be found. The FH69 Blueprint can be considered, in effect, one such complex representation. There have, of course, been many attempts at lumped representations of the catchment as control volume in the past, with more or less physical basis, but with demonstrated success in practical prediction (at least after calibration).

A catchment scale description might not be adequate, however, if the application requires spatially distributed predictions or predictions that reflect the effects of spatially distributed change in the catchment. Where distributed predictions are required, this line of argument would therefore appear to lead towards the use of subdivisions of the catchment into units with similar functioning, or that are, in some sense, hydrologically similar. A crucial question in this context is then: if every point in the catchment has its own unique characteristics how can different points be hydrologically similar? We will return to that question later.

\section{The theory of Reggiani et al. (1999) as an Alternative Blueprint}

A recent highly ambitious attempt to reformulate the theory of hydrological systems in a formal way, that takes explicit account of scale dependencies is due to Reggiani et al. $(1998,1999,2000)$. They formulate conservation equations for mass, energy and momentum and invoke additional entropy constraints for different process sub-domains within an arbitrary hydrological sub-unit of the landscape that they call a "representative elementary watershed" (REW). They define the REW as essentially the area draining to a link in the channel network, although it would seem possible to extend this definition to larger sub-catchment units. Their formulation, after a series of simplifying assumptions, leads to a determinate system of 13 nonlinear coupled balance equations plus 10 geometric relationships in as many unknowns (further work to include evapotranspiration processes within the same framework remains to be done). 
Their work serves as an Alternative Blueprint to $\mathrm{FH} 69$ in its own right. One of the most important aspects of their development is that it is derived directly at the scale of the REW. This approach, therefore, is not based on a direct extension of small scale theory to the larger scale that is implicit in FH69. The equations are derived and solved at the REW scale, as demonstrated in non-dimensional form in Reggiani et al. (2000).

However, it is important to recognise that the balance equations alone are indeterminate (10 coupled equations in 16 unknowns), and that the additional simplifying assumptions are crucial to the closure of the system. Of these additional assumptions, the most crucial are the constitutive relationships for the fluxes within and between REW units (Reggiani et al., 1999). The assumptions made in their initial formulations are that for unsaturated and saturated zone flows, frictional energy losses are a linear function of velocity, while for overland and channel flow, frictional energy losses are a function of velocity squared. These are in keeping with the small scale process equations of Darcy's Law for porous medium flow and uniform flow relationships (Manning, Chezy, Darcy-Weisbach) for overland and channel flow. The theory does not depend on these particular constitutive relationships and Reggiani et al. $(1999,2000)$ stress that other forms could be used where more appropriate.

Something is still lacking, however, from this theory as an Alternative Blueprint. The constitutive relationships required for closure involve coefficients (that may be nonlinear time dependent coefficients in the case of some of the sub-domain flow processes) and Reggiani et al. have conspicuously avoided specifying how these coefficients might be identified at the REW scale. The problem is, in effect, analogous to that of the very difficult closure schemes for descriptions of inhomogeneous turbulence where both the scale dependent dissipation of energy with the flow and the pattern of energy dissipation at the boundaries of the flow interact. In fact, the catchment problem may be more difficult because the constitutive relations for different flow processes will be a function of the detailed geometry of flow pathways that will remain unknowable for the foreseeable future. The theory then leads to a difficult identification problem, involving at least one or two coefficients for each sub-domain within each REW within the catchment, when there is no (current) possibility of direct measurement of flux, energy and momentum at the REW scale (or even state variables such as average potentials and total storage in each sub-domain, etc). 


\section{Theory evaluation: what is "physically-based"?}

The aim of this paper is to provide an Alternative Blueprint for a physically-based hydrological response model. This requires that a definition of "physically-based" be understood. 30 years ago, perhaps this was not such a problem. Physically-based should mean derived deductively from established physical principles, as defined by appropriate assumptions and laws. The FH69 model, albeit a gross simplification of the Navier-Stokes equations, is physically-based in this sense. The approach of Reggiani et al. $(1998,1999)$ is also physically-based in this sense. The assumptions and laws of the hydrological scale descriptions might be questioned, shown to be wanting and improved. That is how the science should progress.

Consistency with defined theoretical assumptions, however, is a necessary but not a sufficient condition for accepting that a model is physically-based. Physically-based should also imply consistency with observations. If consistency is not demonstrated the theory, or the nature of its application, must be wanting (or the observations must be in error or only locally representative). The FH69 model, as applied, is often wanting in this respect and is therefore no more physically-based than other possible descriptions based on principles of mass and energy conservation but defined in terms of variables at different (larger) time and space scales. It has also been shown how the approach of Reggiani et al. may be deficient in this respect, in that it is not clear that the coefficients invoked in the closure assumptions are identifiable in any particular application.

It seems to be necessary to differentiate between physically-based in the sense of being based on defined assumptions and theory, and physically-based in the sense of being consistent with observations. The fact that a model may be physically-based in theory but not consistent with observations results primarily from the mismatch in scales between the scale of the observable state variables and the scale of application. This applies to both the FH69 Blueprint, with its assumption that small scale process descriptions can be applied at larger scales, and the recent Reggiani et al. (1999) theory that is formulated at the scale of the REW but requires the identification of constitutive 
relationships for energy losses at the REW scale for which the observations are not currently available.

In catchment hydrology, the need is not so much for a model that is theoretically acceptable, but for a model that is consistent with observations at the scale of interest. The FH69 Blueprint and Reggiani et al. formulation concentrate on the former. The Alternative Blueprint proposed here concentrates on the latter: a physically-based model is one that is acceptably consistent with the data. It is important to note that this does not necessarily include all models that are consistent with the observational data alone. We would not normally consider as acceptable a model that violated physical principles (albeit as an approximation to the true physics). We may also have evidence or understanding that a model may produce good predictions for what, we believe, are the wrong reasons. In fact, considered honestly, the problem is that this is surely true for all the available models, relative to our qualitative understanding of the processes involved.

Thus, given the limitations of current quantitative hydrological knowledge, this allows for a wide range of potentially acceptable models in defining the model space (the equifinality problem of Beven, 1993, 1996a,b). A valid starting point, in fact, is a model space that comprises of all models that predict the variables of interest (figure 1). This might include many of the so-called conceptual models in hydrology, in so far as they can be shown to be acceptable in not violating physical principles or being inconsistent with available observations. If the interest is only in discharge prediction at the catchment outlet, therefore, the model space will be larger than if distributed predictions of soil moisture and water tables levels are required. It will usually be possible to refine this space by a priori argument (including the classic, if occasionally false, argument that your own model must be better than all other models).

\section{An Alternative Blueprint as method}

So, if there are potentially many models that can be considered as "physically-based" in the sense used here of both not obviously violating physical principles at the scale of application and being consistent with the observational data available, what is being suggested by the previous discussion? There clearly can be no single Alternative Blueprint, but perhaps there can be a formal method of reasoning to arrive at a 
physically-based model, or at least eliminate those possibilities that should not be considered physically-based. The Alternative Blueprint as method can be stated succinctly in terms of the following six stages (see Figure 2):

(i) Define the range of model structures to be considered

(ii) Reject any model structures that cannot be justified as physically feasible a priori for the catchment of interest

(iii) Define the range for each parameter in each model

(iv) Reject any parameter combinations that cannot be justified as physically feasible a priori

(v) Compare the predictions of each potential model with the available observed data (which may include both discharge and internal state measurements) and reject any models which produce unacceptable predictions, taking account of estimated error in the observations.

(vi) Make the desired predictions with the remaining successful models to estimate the risk of possible outcomes.

This methodology has much in common with the Generalised Likelihood Uncertainty Estimation (GLUE) of Beven and Binley (1992, see also Beven et al., 2000; Beven and Freer, 2000b; Beven, 2001). GLUE samples the population of potential models by Monte Carlo simulation and adds a stage of weighting the predictions of each successful model by a likelihood measure in assessing a cumulative distribution of possible outcomes, where the likelihood measure depends on how well a model has done in predicting observed data in the past. This is, however, just one possible implementation of the six stages of this Alternative Model Blueprint.

In Stage (i), defining the feasible model structures is the first stage in defining the "model space" (Figure 1). The FH69 Blueprint is one feasible model structure, that might still be considered for inclusion despite the evidence as to why it should not be considered physically-based in the sense used here, or are there indeed catchments for which its simplifying assumptions are valid? In most catchments, this evidence would be a reason for rejection at Stage (ii) but, as pointed out above, it is probable that all the currently available models could be rejected for one good physical reason or another. Since it is not yet possible to describe either flow structures or parameter values unambiguously at 
the scales of interest, there is potential for a number of competing models at Stage (i). This could include, for example, different sets of closure assumptions within the Reggiani et al. (1999) theory.

Stage (iii) completes the definition of the model space but in that it is ranges of effective parameter values that are required in every model under consideration, then it may not be easy to decide what ranges to specify in Stage (iii), or which combinations of parameter values (parts of the model space) to reject in Stage (iv). This decision will commonly be left to the evaluation of Stage (v) which will determine the set of successful models. For ungauged catchments Stages ( $v$ ) cannot be implemented, making the choice of feasible parameter sets at Stage (iv) that much more important.

Note: that there is no mention of optimisation of models in this methodology. It is therefore consistent with the evidence of equifinality of models that has come from many explorations of different model spaces (see, for example, Beven et al., 2000). This does, however, create a problem of searching the defined model space for the set of models that are feasible. For model spaces of small dimension, this is not a great problem even for some complex models, using Monte Carlo, structured Monte Carlo or discrete sampling strategies. For large model spaces (many model structures with multiple or distributed parameters) it is a very real practical problem. It is a problem that has been somewhat mitigated for many problems by the development of parallel computing systems that enable large numbers of model runs to be made relatively cheaply and quickly. However, reliance must still be made on an assumption that a sufficient sample of successful models from the model space have been considered to give an appropriate range of predicted outcomes. At present, implementation of this Alternative Blueprint will not be possible for the most complex distributed hydrological models (although experiments are already being carried out with the SHE model and with a distributed groundwater transport model).

\section{Equifinality, theory falsification and hypothesis testing in hydrology}

My argument thus far is therefore that there may be many models of the catchment hydrological system that are physically-based in the sense that they do not violate physical principles and that may be consistent with the limited observables. Qualitative 
understanding of small scale hydrological processes is not the problem here, it is the implementation of that understanding at larger scales to specific, unique, catchments that leads to many different acceptable modelling possibilities. This type of modelling equifinality is a generic problem. It would be a problem even in the case where we had a "true" model description but had to identify appropriate parameter sets for a specific site given only limited observational information. There will still be many parameter sets that would prove to be consistent with the data available.

In one sense, the problem of equifinality can be considered a problem of decidability. We will only be forced to accept that there must be multiple model representations of hydrological systems if we cannot decide between them in some way, other than the traditional, but rather arbitrary, practice of only considering, a priori, one model and one "optimal" set of parameters in that model. If we could decide that one set of models is not an adequate description of the system under study, then clearly we should be rejecting that set of models. Theoretical and observational limitations may make such decisions difficult but, in principle, it is possible to work within such a falsificationist framework.

The problems of falsification and decidability are traditional problems in science and have been the subject of considerable philosophical discussion. Note that it is not being suggested that any large scale hydrological descriptions need be considered as true descriptions of reality. That must surely be considered as currently impossible in modelling most open environmental systems. We can therefore avoid many of the philosophical criticisms of crude falsification (as outlined in a hydrological context, for example, by Oreskes et al., 1994). In fact, the approach we are suggesting is much more a relativistic approach, within which some of the feasible models are considered to be more likely than others but where falsification can be used as a means of reducing the range of possibilities. Thus, given multiple working hypotheses (competing models) what is the best decidable hypothesis test to differentiate between them and reject some of the multiple possibilities? A critical test is potentially a very effective constraint in reducing the set of feasible models but not all the hypotheses that we could pose will be testable given the observational techniques available. 
In the past history of science, of course, there have some hypotheses posed on the basis of deductive reasoning that have not been testable until appropriate observational techniques have been developed. This has been a significant encouragement for the development of measurement technology. Could the same be true in hydrology? Or, as has been the case in many other subject areas, will the development of better theory follow the development of new measurement techniques?

Are there valuable hypotheses that are not yet testable in hydrology? Unfortunately, since we cannot even firmly close the areal water balance equation in hydrology, the answer is yes, even for the most fundamental hypotheses. In hydrological modelling, one of the most important hypotheses to test would have to be the assumed nature of the fluxes between control volumes under different conditions. This would require either a direct measurement technique for water fluxes at the chosen control volume scale, or measurements of water storage for the control volume as a whole or as different component storages, together with the boundary conditions, from which the magnitudes of the inter-volume fluxes could be (approximately) inferred. These measurements cannot currently be made at the scales required (with the exception of catchment discharge measurements where the control volume is at the catchment scale, but other catchment scale boundary fluxes, especially evapotranspiration from a control volume cannot easily be measured).

Are there more limited hypotheses that can be tested using currently available measurement techniques? That macropore fluxes are important at the control volume (or REW) scale? That the fast response of a catchment is dominated by infiltration excess overland flow? That energy losses for subsurface flows are a linear function of an effective mean velocity in a heterogeneous soil? That energy losses for surface flows are a function of an effective mean velocity squared on a complex surface topography? That valley bottom storage plays an important role in shaping surface hydrographs? That recharges to groundwaters are dominated by short periods of high fluxes? Many hypotheses can be envisaged (though not all easily testable) that would have value in this type of evaluation context. 


\section{Conclusions: Mathematical complexity and observational capability}

There is a certain prestige in science associated with the use of complex mathematics. Difficult sciences, such as biology, ecology, hydrology and other environmental sciences often aspire to demonstrate progress and maturity by more advanced mathematical descriptions. Until the FH69 Blueprint, it had not been necessary for hydrologists to worry much about partial differential equations and their analytical and numerical solution, if only because of computational constraints. This was still the era of using electrical analogue computers for the solution of the Laplace equation as an approximate description of steady state groundwater flow (see IAHS, 1968).

Computing constraints are now much less critical for many problems of great practical interest, but with hindsight there would appear to be more important problems in theoretical hydrology than increasing mathematical complexity. This is not to deny that physical descriptions of hydrological processes might not need to be complex but mathematical complexity can also imply constraint. The FH69 Blueprint is constrained by its assumptions to a Darcian description of subsurface flow. A more general Darcian description of flow in soil with heterogeneous characteristics will be constrained by the assumptions made about the nature of the heterogeneity (and introduces additional parameters to be determined). Unless those assumptions can be shown independently to be valid, within the context of uniqueness of place and the limitations of available data, they may result in inappropriate constraints on predictions.

Evaluation of assumptions, regardless of mathematical complexity, is then a matter of observational capability. It is therefore appropriate that we should return at the end of this description of an Alternative Blueprint as method, to a focus on observation. To state a need for improved observational capability is not new, but the Alternative Blueprint that has been outlined here has an explicit role for observation in conditioning the model space and model rejection that was lacking in the FH69 version. There, prior assumption and theory was emphasised. The Alternative Blueprint has been presented here with essentially no equations. The exposition of the mass continuity equation serves only to highlight some of the problems encountered in defining a physically-based model at the scales required. It is, however, no less an "artist's conception" of a 
Blueprint for a way of working than was the more mathematical Freeze and Harlan version 30 years ago.

Physically-based theory must be consistent with observations. In this sense the FH69, as applied in current models, is often not consistent with process observations and is not therefore physically-based. In a science where unique local characteristics so affect local observations as to make the application of small scale physical theory intractable at the larger scale, we must look to alternative larger scale descriptions and must recognise that there may be many possible descriptions that provide equally good predictions of the observations. The real challenge is to find creative ways of using observations to limit those possibilities. The Alternative Blueprint described here provides one way of formalising that process.

\section{References}

Abbot, M. B., Bathurst, J. C., Cunge, J. A., O'Connell, P. E. and Rasmussen, J., 1986, An introduction to the European Hydrological System - Système Hydrologique Européen, SHE. 2. Structure of a physically-based, distributed modelling system., Journal of Hydrology, 87-77.

Abbott, M. B. and Refsgaard, J-C., 1996, Distributed Hydrological Modelling, Kluwer Academic, Dordrecht

Ambroise, B., K.J. Beven and J. Freer, 1996, Towards a generalisation of the TOPMODEL concepts: topographic indices of hydrological similarity, Water Resources Research, 32(7), 2135-2145.

Barling, R. D., Moore, I. D. and Grayson, R. B., 1994, A quasi-dynamic wetness index for characterising the spatial distribution of zones of surface saturation and soil water content, Wat. Resour. Res., 30, 1029-1044.

Bathurst, J. C., Wicks, J. M. and O'Connell, P. E.,. 1995, The \{SHE/SHESED\} Basin Scale Water Flow and Sediment Transport Modelling System, in Singh, V. P., Computer Models of Watershed Hydrology, 563-594, Water Resource Publications, Highlands Ranch, Co. 
Beven, K.J., 1989, Changing ideas in hydrology: the case of physically based models. J. Hydrology, 105, 157-172.

Beven, K. J., 1993, Prophecy, reality and uncertainty in distributed hydrological modelling, Adv. in Water Resourc., 16, 41-51

Beven, K.J., 1995, Linking parameters across scales: sub-grid parameterisations and scale dependent hydrological models, Hydrological Processes, 9, 507-526.

Beven, K.J., 1996a, Equifinality and Uncertainty in Geomorphological Modelling, In B L Rhoads and C E Thorn (Eds.), The Scientific Nature of Geomorphology, Wiley, Chichester, 289-313.

Beven, K.J., 1996b, A discussion of distributed modelling, Chapter 13A, In J-C Refsgaard and M B Abbott (Eds.) Distributed Hydrological Modelling, Kluwer, Dordrecht, 255-278.

Beven, K J, 1997, TOPMODEL: a critique, Hydrological Processes, 11(3), 1069-1085.

Beven, K. J., 2000, Uniqueness of place and the representation of hydrological processes, Hydrology and Earth System Science, 4(2), 203-213.

Beven, K. J., 2001, Rainfall-Runoff Modelling - The Primer, Wiley, Chichester.

Beven, K.J., Kirkby, M.J., 1979, A physically-based variable contributing area model of basin hydrology. Hydrological Sciences Bulletin, 24(1), 43-69.

Beven, K.J., R. Lamb, P. Quinn, R. Romanowicz and J. Freer, 1995, TOPMODEL, in V.P. Singh (Ed.) Computer Models of Watershed Hydrology, Water Resource Publications, Colorado, 627-668.

Beven, K J and Franks, S W, 1999, Functional similarity in landscape scale SVAT modelling, Hydrology and Earth Systems Science, 3(1), 85-94.

Beven, K. J. and Freer, J., 2000a, A dynamic TOPMDEL, Hydrological Processes, in press.

Beven, K. J. and Freer, J., 2000b, Equifinality, data assimilation, and uncertainty estimation in mechanistic modelling of complex environmental systems, J. Hydrology, in press.

Beven, K J, Freer, J, Hankin, B and Schulz, K, 2000, The use of generalised likelihood measures for uncertainty estimation in high order models of environmental systems. 
Nonlinear and Nonstationary Signal Processing, W J Fitzgerald, R L Smith, A T Walden and P C Young (Eds). CUP, in press

Binley, A.M., Beven, K.J., Elgy, J., 1989, A physically-based model of heterogeneous hillslopes. II. Effective hydraulic conductivities. Water Resources Research, 25(6), 1227-1233.

Bronstert, A. and Plate, E., 1997, Modelling of Runoff Generation and Soil Moisture Dynamics for Hillslopes and Microcatchments, J. Hydrology, 198, 177-195

Calver, A. and Wood, W. L., 1995, The Institute of Hydrology Distributed Model, in V.P. Singh (Ed.) Computer Models of Watershed Hydrology, Water Resource Publications, Colorado, 595-626.

Dagan, G, 1986, Statistical theory of groundwater flow and transport: pore to laboratory; laboratory to formation and formation to regional scale, Wat. Resour. Res., 22, 120135.

De Marsily, G, 1994, Quelques réflexions sur l'utilisation des modèles en hydrologie, Revue des Sciences de l'Eau, 7(3), 219-234.

Duffy, C. J., 1996, A Two-State Integral-Balance Model for Soil Moisture and Groundwater Dynamics in Complex Terrain, Water Resources Research, 32, 24212434

Eagleson, P, 1970, Dynamic Hydrology, McGraw-Hill, New York

Ewen, J., 1996, SAMP model for water and solute movement in unsaturated porous media involving thermodynamic subsystems and moving packets. 1. Theory, J. Hydrology, 182, 175-194.

Faeh, A. O., Scherrer, S. and Naef, F.,1997,A Combined Field and Numerical Approach to Investigate Flow Processes in Natural Macroporous Soils under Extreme Precipitation, Hydrology and Earth System Sciences, 1(4), 787-800,

Franks, S and Beven, K J, 1997, Estimation of evapotranspiration at the landscape scale: a fuzzy disaggregation approach, Water Resour. Res., 33(12), 2929-2938.

Freeze, R. A., 1972, Role of Subsurface Flow in Generating Surface Runoff. 2. Upstream Source Areas, Water Resources Research, 8(5), 1272-1283 
Freeze, R. A. and Harlan, R. L., 1969, Blueprint for a physically-based, digitallysimulated hydrologic response model, J. Hydrol., 9, 237-258.

Germann, P. F. and Di Pietro, L., 1999, Scales and dimensions of momentum dissipation during preferential flow in soils, Water Resources Research, 35(5),14431454.

Grayson, R. B. and Moore, I. D. and McMahon, T. A., 1992, Physically-based hydrologic modelling. 2. Is the concept realistic?, Water Resources Research, 28, 2659

IAHS, 1968, Symposium on the use of analogue and digital computers in hydrology, Int. Assoc. Sci. Hydrol./UNESCO Pubn. No. 80.

Jakeman, A., and Hornberger, G. M.,1993, How much complexity is warranted in a rainfall-runoff model?, Water Resources Research, 29, 8, 2637-2649

Jaynes, D. B., 1990, Soil Water Hysteresis: Models and Implications, in Anderson, M. G. and Burt, T. P., Process Studies in Hillslope Hydrology, Wiley, Chichester, UK, 93126.

Lamb, R., Beven, K. J. and Myrabø, S., 1997, Discharge and water table predictions using a generalised TOPMODEL formulation, Hydrol. Process., 11(9), 1145-1168

Levi, E., 1995, The Science of Water: The Foundations of Modern Hydraulics, American Society of Civil Engineers, New York, 649pp

Loague, K. M. and Kyriakidis, P. C., 1997, Spatial and temporal variability in the R-5 infiltration data set: déjà vu and rainfall-runoff simulations, Water Resources Research, 33, 2883-2896

Neuman, S. P., 1995, On advective transport in fractal permeability and velocity fields, Water Resources Research, 31, 1455

Oreskes, N., Schrader-Frechette, K. and Belitz, K., 1994, Verification, Validation and Confirmation of Numerical Models in the Earth Sciences, Science, 263, 641-646

Pachepsky, Y. and Timlin, D., 1998, Water transport in soils as in fractal media, J. Hydrology, 204, 98-107

Parkin, G., O'Donnell, G., Ewen, J., Bathurst, J. C., O'Connell, P. E., and Lavabre, J., 1996, Validation of catchment models for predicting land-use and climate change impacts. 2. Case study for a Mediterranean catchment, J. Hydrology, 175, 595-613 
Prigogine, I, 1997, The End of Certainty, The Free Press, New York.

Refsgaard, J-C. and Storm, B., 1995. MIKE SHE, in Singh, V. P, Computer Models of Watershed Hydrology, Water Resources Publications, Highlands Park, Co., 809-846

Reggiani, P., Sivapalan, M and Hassanizadeh, S. M., 1998, A unifying framework for watershed thermodynamics: balance equations for mass, momentum, energy and entropy and the second law of thermodynamics, Advances in Water Resources, 23(1), 15-40.

Reggiani, P., Hassanizadeh, S. M., Sivapalan, M. and Gray, W. G., 1999, A unifying framework for watershed thermodynamics: constitutive relationships, Advances in Water Resources, 23(1), 15-40.

Reggiani, P., Sivapalan, M and Hassanizadeh, S. M., 2000, Conservation equations governing hillslope responses: exploring the physical basis of water balance, water Resources Research, 36, 1845-1863.

Seibert, J., 1997, Estimation of Parameter Uncertainty in the HBV Model, Nordic Hydrology, 28, 247-262

Singh, V. P., 1996, Kinematic Wave Modelling in Water Resources, Wiley, Chichester

Smith, R. E., Goodrich, D. R., Woolhiser, D. A. and Simanton, J. R., 1994, Comments on "Physically-based hydrologic modelling. 2. Is the concept realistic?" by Grayson, R. B. and Moore, I. D. and McMahon, T. A., Water Resources Research, 30, 851-854.

Stephenson, R. and Freeze, R. A., 1974, Mathematical Simulation of Subsurface Flow Contributions to Snowmelt Runoff, Reynolds Creek, Idaho, Water Resources Research, 10(2), 284-298.

Vertessy, R. A., Hatton, T. J., O'Shaughnessy, P. J. and Jayasuriya, M. D. A., 1993, Predicting Water Yield from a Mountain Ash Forest Using a Terrain Analysis Based Catchment Model, J. Hydrology, 150, 665-700

Woolhiser, D. A., 1996, Search for physically-based runoff model - a hydrologic El Dorado, J. Hydraul. Eng. ASCE, 122, 122-129

Young, P.C. and K.J. Beven 1994, Data-based mechanistic modelling and the rainfallflow non-linearity, Environmetrics, V.5, 335-363. 


\section{Figure Headings}

Figure 1 The Model Space. Note that model structure (Model 1, Model 2, ...Model N) acts as one dimension of choice. $\mathrm{P}_{1}, \mathrm{P}_{2}, \mathrm{P}_{3}$, represent 3 of perhaps many parameter dimensions. There may be different dimensions for different model structures but each model should predict all variables of interest (if not, this may provide one justification for rejection).

Figure 2 Stages in the Alternative Blueprint for Physically-Based Modelling. 